\title{
Terahertz active imaging radar: preprocessing and experiment results
}

\author{
Gang Yao ${ }^{*}$ and Yiming Pi
}

\begin{abstract}
A terahertz $(\mathrm{THz})$ radar provides the possibility of higher precision imaging due to the wider bandwidth. A summary of a THz imaging radar system is presented with emphasis on $\mathrm{THz}$ radar component design, system design, and detective imaging. In this article, we introduce a linear frequency-modulated continuous wave (LFMCW) radar system with a 4.8-GHz bandwidth and theoretical resolution of $3.125 \mathrm{~cm}$. The heterodyne RF receiver structure is applied to the system to reduce the sampling rate. A non-linear correction method is applied to compensate the range backscatter signal. With the presented LFMCW radar system, high-resolution images $(3.5 \mathrm{~cm} \times 3.5 \mathrm{~cm})$ are achieved using the ISAR imaging technique. The experiments performed on the real LFMCW radar data have shown the capability of high-resolution imaging.
\end{abstract}

Keywords: Terahertz; LFMCW radar; Error correction; ISAR

\section{Introduction}

A terahertz frequency band is a very important research and valuable undeveloped frequency resource, which especially has a great potential for the development of a high-resolution imaging radar. Compared to the traditional microwave radar, the advantages of a terahertz radar system are as follows: Firstly, the shorter wavelength is favorable toward providing a wider bandwidth, which could benefit the higher precision of imaging. Secondly, the narrow antenna beam in the terahertz band could not only obtain higher antenna gain in radar LOS, improving the ability of multi-target discrimination and recognition, but also reduce the opportunity of main lobe jamming [1-3]. The terahertz radar detection system is an important direction of terahertz technology domestic and overseas [4].

A linear frequency-modulated continuous wave (LFMCW) radar has advantages of high range resolution, low transmit power, high receiver sensitivity, simple structure, etc. $[5,6]$. There is no distance blind area, better anti-stealth ability than a pulse radar, anti-background clutter, and antijamming characteristics, and it is particularly suitable for near-range applications [7]. A terahertz wave has great

\footnotetext{
* Correspondence: yaog@vip.sina.com

School of Electronic Engineering, University of Electronic Science and Technology of China, Chengdu 611731, China
}

bandwidth itself, so making use of the LFMCW radar structure can obtain a very high range resolution; the emission power of the terahertz wave is still very low at present, and the LFMCW radar has lower emission power than the pulse radar, so using a LFMCW radar system can reduce the transmitter power requirements. In consideration of the great bandwidth of terahertz waves and the high range resolution of the LFMCW radar, the terahertz LFMCW radar can obtain high range resolution $[8,9]$.

Currently, the international research institutions with terahertz radar experimental systems are the Jet Propulsion Laboratory (JPL) $(0.6 \mathrm{THz}$ radar imaging system) [10] and the German Institute of Applied Science (Forschungsgesellschaft für Angewandte Naturwissenschaften, FGAN) High Frequency Physics and Radar Techniques (FHR) Laboratory $(0.22 \mathrm{THz}$ COBRA inverse synthetic aperture radar (ISAR) imaging system) [11]. In 2006, RJ Dengler and KB Cooper et al. of JPL have successfully developed the first highresolution terahertz imaging system with a $2-\mathrm{cm}$ range resolution; this system introduced FMCW radar technology into the imaging system, processed the waveform distortion compensation by software, and obtained a 2 $\mathrm{cm}$ range resolution (internal $4 \mathrm{~m}$ ) [12]. In 2008, KB Cooper et al. who came up with a $0.6 \mathrm{THz}$ radar imaging system have successfully developed a $0.58 \mathrm{THz}$ high-resolution three-dimensional imaging radar system

\section{实}

(c) 2014 Yao and Pi; licensee Springer. This is an open access article distributed under the terms of the Creative Commons Attribution License (http://creativecommons.org/licenses/by/2.0), which permits unrestricted use, distribution, and reproduction in any medium, provided the original work is properly cited. 


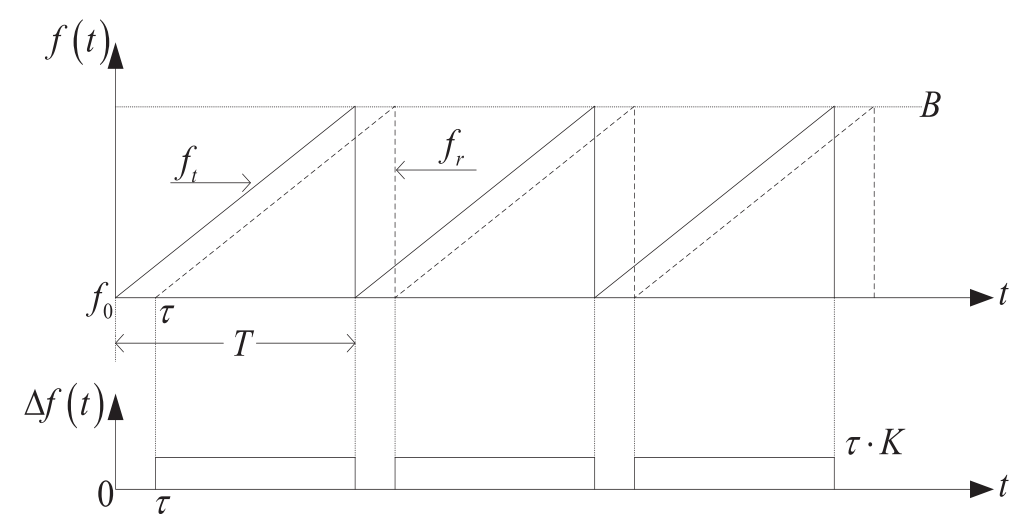

Figure 1 Time-frequency of transmitting signal $\left(f_{t}\right)$, receiving signal $\left(f_{r}\right)$, and beat signal $(\Delta f(t))$.

based on the $0.6 \mathrm{THz}$ radar imaging system [13]. The imaging system used in ISAR imaging can obtain subcentimeter resolution. In 2007, Essen and Wahlen et al. of the German Institute of Applied Science (FGAN) High Frequency Physics and Radar Technology (FHR) Laboratory have successfully developed a 220-GHz terahertz imaging radar system COBRA-220 [14]. The system is also based on a LFMCW radar system, in which the FM bandwidth is $8 \mathrm{GHz}$, successfully achieving the $1.8-\mathrm{cm}$ range resolution in a $200-\mathrm{m}$ distance.

In this paper, we present an overview of the $\mathrm{THz}$ imaging radar technology. The radar is currently a portable laboratory prototype system operating in a LFMCW mode over a $4.8-\mathrm{GHz}$ bandwidth in the University of Electronic Science and Technology of China (UESTC). The remainder of this paper is organized as follows. Section 2 describes the LFMCW signal model. In Section 3, a dual-source structure model for an intermediate frequency receiver in the $\mathrm{THz}$ radar is developed. This is followed by Section 4 in which a detailed analysis of the signal and the correcting method are presented. In Section 5, the experiment results for the LMFCW THz radar are shown. We conclude the paper in Section 6.

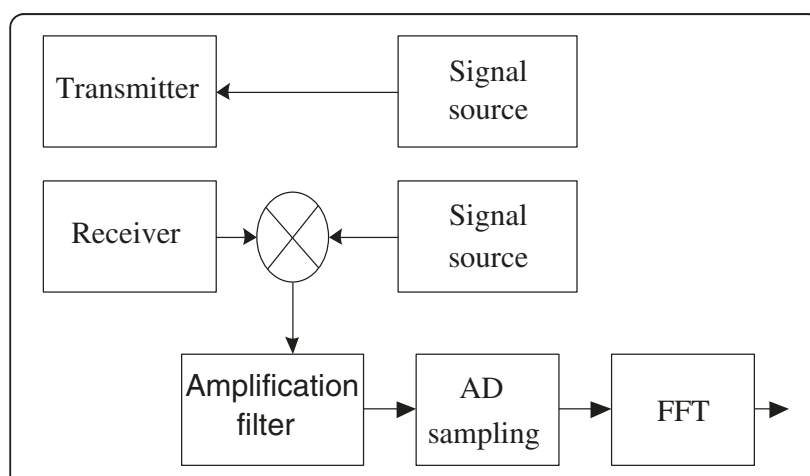

Figure 2 The conventional LFMCW radar system structure.

\section{LFMCW signal analysis}

In ideal conditions, the LFMCW radar's transmitting and receiving signal frequency can be shown in the Figure 1. The transmitting signal is expressed as

$$
S_{t}(t)=\exp \left\{2 \pi f_{0} t+\pi K t^{2}+\theta_{0}\right\}, 0 \leq t \leq T
$$

where $f_{0}$ is the carrier frequency, $t$ is the time variable, $K$ is the frequency modulation slope, $T$ is the duration of the signal, and $\theta_{0}$ is the initial phase.

If $\tau$ is the time delay of a stationary target at the range $R$, then the received signal can be expressed as

$$
\begin{aligned}
S_{r}(t) & =S_{t}(t-\tau) \\
& =\exp \left\{2 \pi f_{0}(t-\tau)+\pi K(t-\tau)^{2}+\theta_{0}\right\}, \tau \leq t \leq T
\end{aligned}
$$

where $\tau=2 R / c$, and $c$ is the speed of light.

In order to reduce the sampling rate, the beat signal can be obtained in intermediate frequency after mixing the transmitting signal and the received echo signal.

$$
\begin{aligned}
S_{b}(t) & =S_{t}(t) \times S_{r}{ }^{*}(t) \\
& =\exp \left\{2 \pi f_{0} \tau+2 \pi K \tau t-\pi K \tau^{2}\right\}, \tau \leq t \leq T
\end{aligned}
$$

The beat frequency can be calculated which is the derivative of the beat signal phase.

$$
f_{b}(t)=\frac{1}{2 \pi} \times \frac{d \Phi_{b}(t)}{d t}=K \tau=K \frac{2 R}{c}
$$

It is obvious that the beat frequency is a fixed value, only related to the distance and frequency modulation slope. Then, the range of target is expressed as

$$
R=\frac{f_{b} c}{2 K}=\frac{c T}{2 B} f_{b}
$$

So the frequency of the beat signal is the identification to the target range. After sampling was carried out on the beat signal of the LFMCW radar, the beat frequency signal's frequency spectrum can be obtained by discrete 


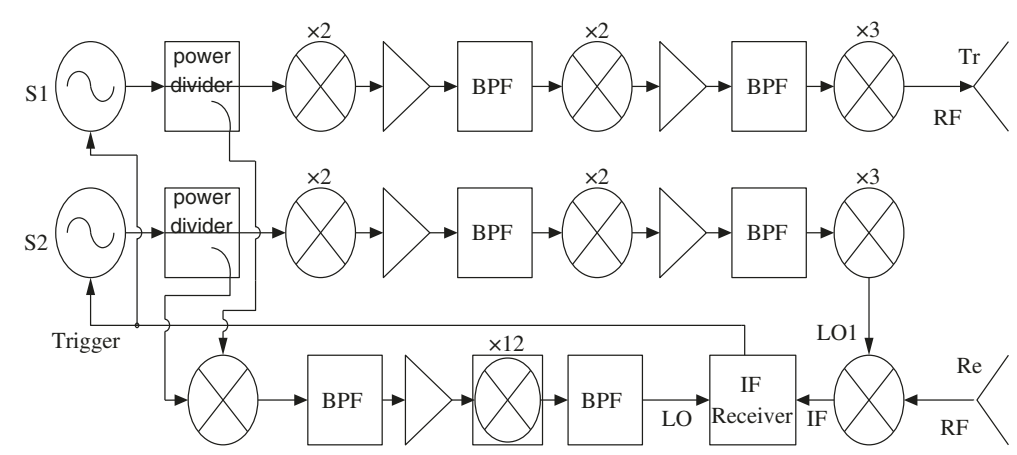

Figure $3 \mathrm{THz}$ LFMCW radar system structure.

Fourier transform (DFT). In the spectrum, the peak lines are corresponding to the beat frequency and static target's range.

\section{Intermediate frequency receiver}

Figure 2 can show the conventional LFMCW radar's system structure from which the signal model is analyzed in previous chapters. The power of the signal source is limited by the apparatus in the $\mathrm{THz}$ wave band. The single frequency source has difficulty transmitting and mixing at the same time. If two independent frequency sources are employed in a radar system, it may lead to the issue of phase out of sync.

The 'non-coherent dual-source implementing coherence' way is applied in the THz LFMCW radar system. The coherent system structure can be shown in Figure 3. Mixing twice needs to be done in order to realize the coherent system. The intermediate frequency receiver is designed after the first mixing of the received $\mathrm{THz}$ band frequency signal. The second mixing, I/Q demodulation, signal correction, and some other processing are involved in the intermediate frequency receiver.
Signal sources $S_{1}$ and $S_{2}$, two low-frequency linear frequency modulation signals, are used to produce the transmitting signal and the local oscillator (LO) signal, respectively. They can be respectively represented as

$$
\begin{aligned}
& S_{1}(t)=A_{1} \exp \left\{2 \pi f_{C 1} t+\pi K_{S 1} t^{2}+\phi_{1}\right\} \\
& S_{2}(t)=A_{2} \exp \left\{2 \pi f_{C 2} t+\pi K_{S 2} t^{2}+\phi_{2}\right\}
\end{aligned}
$$

where $f_{c 1}$ and $f_{c 2}$ are the carrier frequency, $t$ is the time variable, $K_{s 1}$ and $K_{s 2}$ are the frequency modulation slope, and $\phi_{1}$ and $\phi_{2}$ are the initial phase.

The transmitting signal can be generated from the source $S_{1}$ by means of $\times 12$ frequency multiplication $(\times 2$ twice and $\times 3$ once), band-pass filtering, and amplification. Then, it can be represented as

$$
S_{T}(t)=A_{T} \exp \left\{2 \pi \cdot 12 f_{C 1} t+\pi \cdot 12 K_{0} t^{2}+12 \phi_{1}\right\}
$$

where $A_{T}$ is the signal amplitude. It is supposed that $R(t)$ is the range of target and $\tau(t)=2 R(t) / c$ is the echo signal time delay. The received echo signal can be expressed as

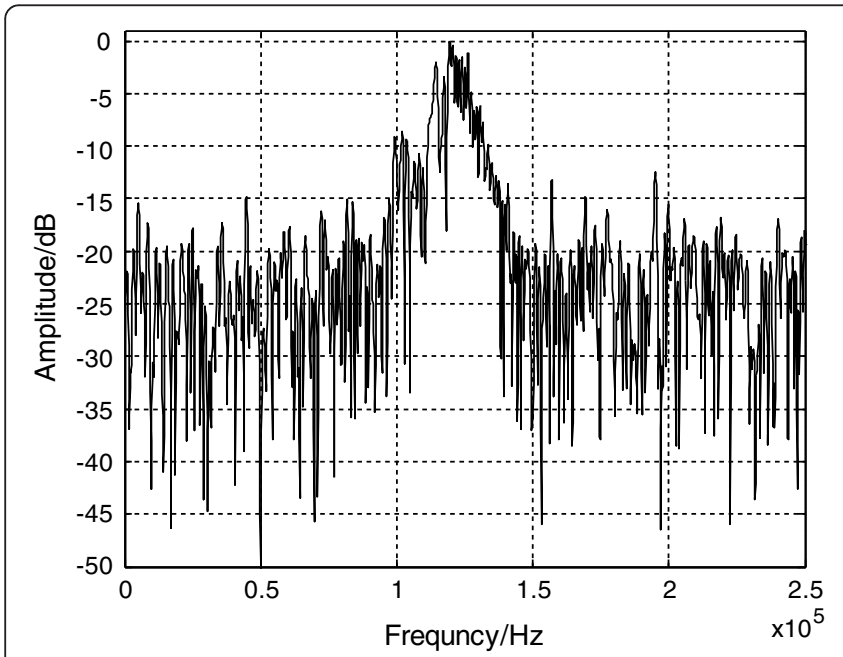

(a)

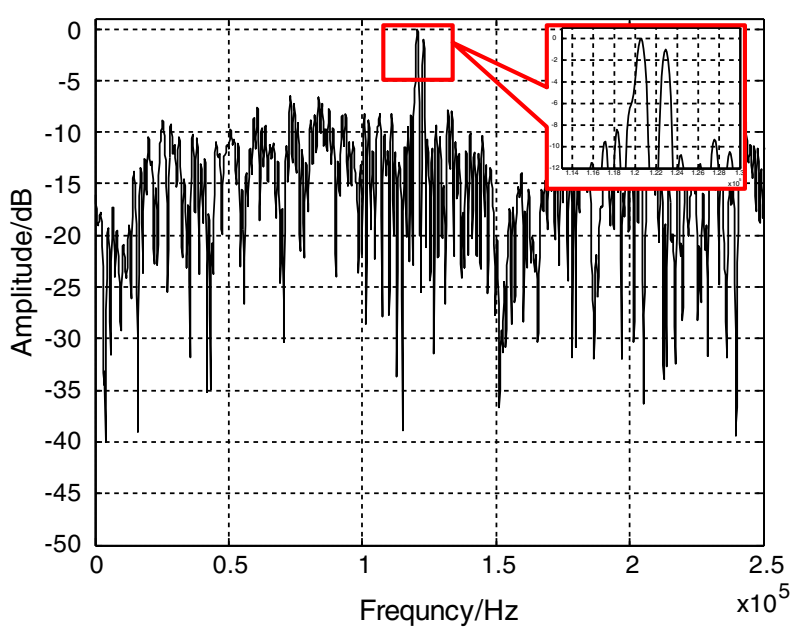

(b)

Figure 4 Range spectrum. (a) The raw range spectrum of two point-like targets. (b) The range spectrum result after compensation. 


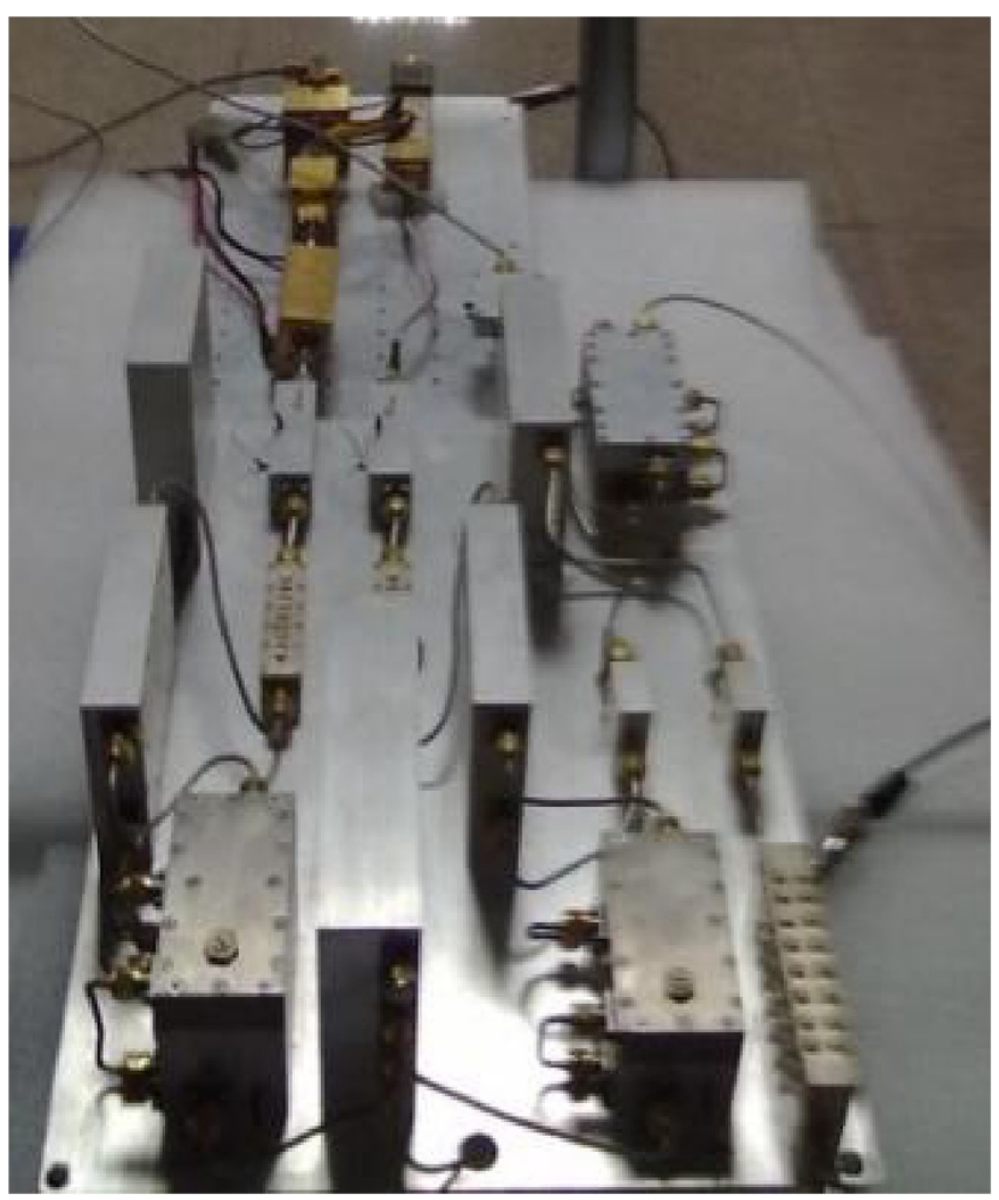

Figure 5 The real product photo of the THz LFMCW radar.

$$
\begin{aligned}
S_{R}(t)= & K A_{T} \exp \left\{2 \pi \cdot 12 f_{C 1}(t-\tau(t))\right. \\
& \left.+\pi \cdot 12 K_{0}(t-\tau(t))^{2}+12 \phi_{1}\right\}
\end{aligned}
$$

The LO signal in the $\mathrm{THz}$ band can come from the source $S_{2}$ by the same procedure as the transmitting signal. It can be expressed as

$$
S_{\mathrm{LO} 1}(t)=A_{\mathrm{LO} 1} \exp \left\{2 \pi \cdot 12 f_{\mathrm{C} 2} t+\pi \cdot 12 K_{0} t^{2}+12 \phi_{2}\right\}
$$

where $A_{\mathrm{LO}}$ is the amplitude of the LO signal. The first mixing is realized between the received echo signal $\left(S_{R}(t)\right)$ and the LO signal $\left(S_{\mathrm{LO} 1}(t)\right)$. So the obtained intermediate signal is

$$
\begin{aligned}
S_{\mathrm{IF}}(t)= & A_{\mathrm{IF}} \exp \left\{2 \pi \left[12\left(f_{C 2}-f_{C 1}\right) t+12 f_{C 1} \tau(t)\right.\right. \\
& \left.\left.+\mu \tau t(t)-\mu \tau(t)^{2} / 2\right]+12 \Delta \phi\right\}
\end{aligned}
$$

where $A_{\mathrm{IF}}$ is the amplitude of the intermediate frequency signal, $\mu=12 K_{0}$ is the frequency modulation slope, and $\Delta \phi=\phi_{2}-\phi_{1}$ is the difference of initial phases.
The other LO signal is acquired through the following procedure: mixing $S_{1}$ and $S_{2}, \times 12$ frequency multiplication, amplifying, and filtering. It can be represented as

$$
S_{\mathrm{LO}}(t)=A_{\mathrm{LO}} \exp \left\{2 \pi \cdot 12\left(f_{\mathrm{C} 2}-f_{\mathrm{C} 1}\right) t+12 \Delta \phi\right\}
$$

In the end, the result of mixing intermediate frequency signal $S_{\mathrm{IF}}(t)$ and LO signal $S_{\mathrm{LO}}(t)$ is

$$
\begin{aligned}
S_{B}(t) & =S_{\mathrm{IF}}(t) \times S_{\mathrm{LO}}^{*}(t) \\
& =A_{B} \exp \left[2 \pi\left(\mu \tau(t) t+12 f_{C 1} \tau(t)-\mu \tau(t)^{2} / 2\right)\right]
\end{aligned}
$$

The results show that the initial phase difference is offset through taking advantage of the dual-source system structure. The results are consistent with the traditional single-source LFMCW radar system structure. Thereby, the problem about the dual-source's non-sync can be solved effectively. 


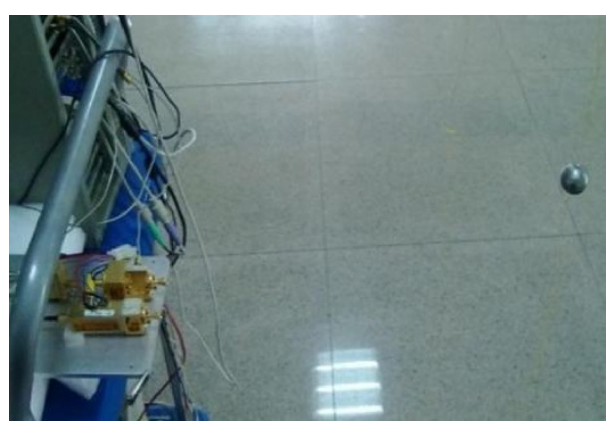

(a)
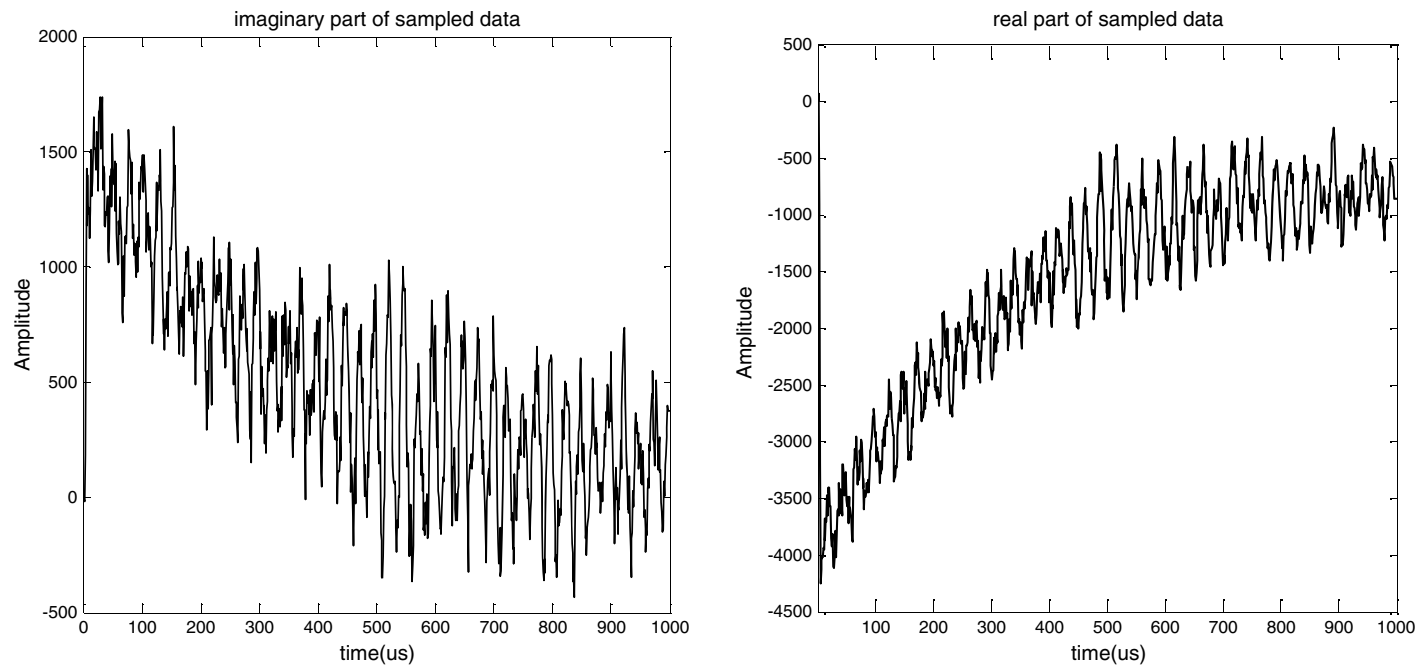

(b)

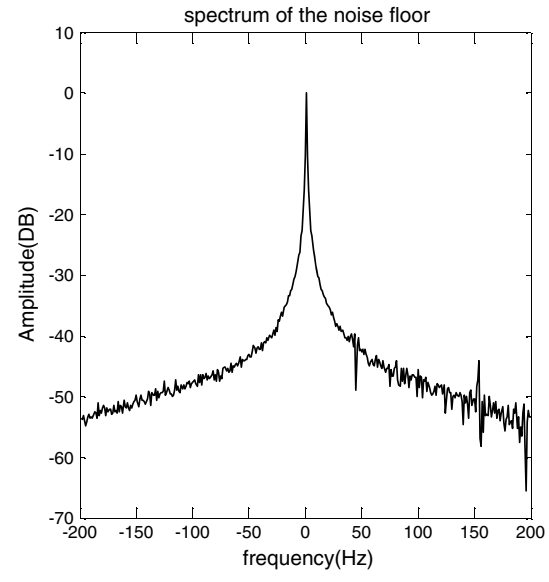

(c)

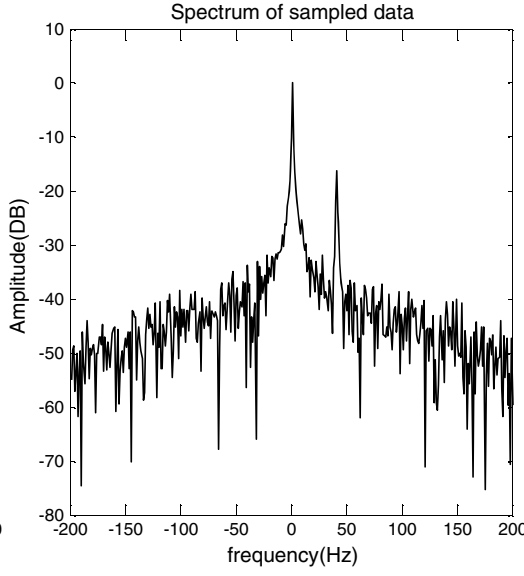

(d)

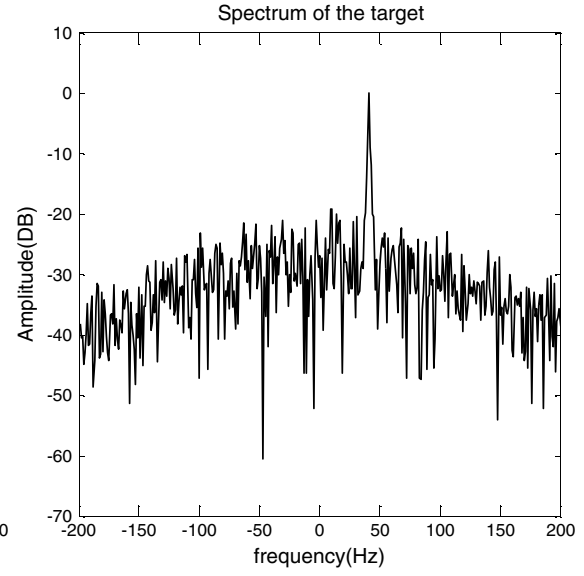

(e)

Figure 6 Metal ball range detection experiment. (a) Single metal ball testing scene. (b) The sampled data (real and imaginary part). (c) Spectrum of the signal noise floor detected. (d) Range detection without error correction. (e) Range detection after error correction (range $1.25 \mathrm{~m}$, frequency $40 \mathrm{kHz}$ ).

\section{Error analysis and correction}

In the FMCW radar, the range information of the target can be obtained from the spectral content of the final signal. The range resolution of a FMCW radar depends only on the bandwidth of the transmitting signal. The range resolution is the minimum distance that two targets 


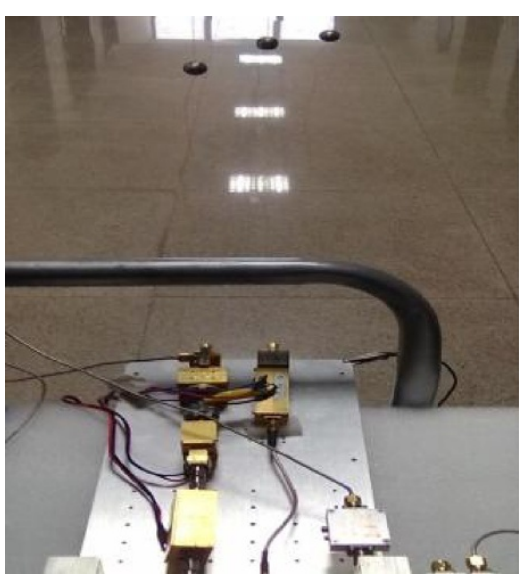

(a)

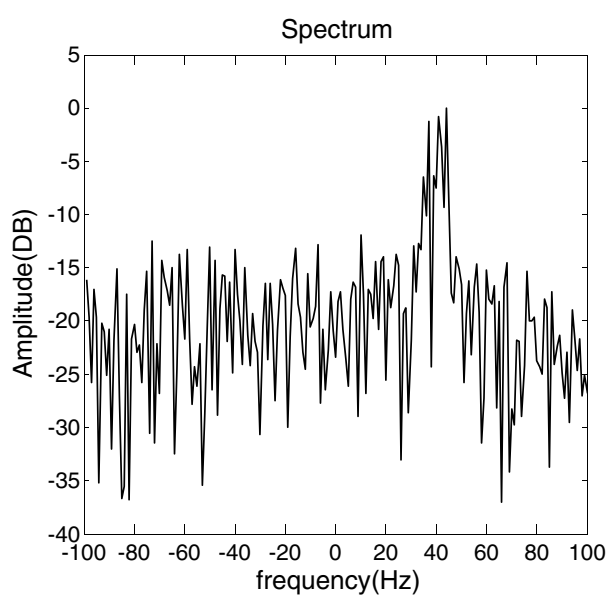

(b)

Figure 7 Testing of three metal balls. (a) Testing scene of three metal balls. (b) The spectrum of sampled data for range detection.

can be separated along the radar's line of sight before they are indistinguishable.

If the radar tone is measured for the time $\Delta t$, the Fourier-limited single-target spectral width will be $\delta f_{b \text { min }}=$ $1 / \Delta t$. In according to formula (5), the minimum range resolution can become

$$
\delta_{r \text { min }}=\frac{c}{2 K_{0}} \delta f_{b \text { min }}=\frac{c}{2 K_{0}} \frac{1}{\Delta t}=\frac{c}{2 \Delta F}
$$

It means that the range resolution only depends on the total swept bandwidth. The inverse relationship between the range resolution and bandwidth is similar to the ordinary pulsed radar. With a bandwidth of $4.8 \mathrm{GHz}$, the theoretical range resolution of our $\mathrm{THz}$ radar is $3.1 \mathrm{~cm}$; however, this theoretical value is only achieved if unwanted modulation in the LFM waveform is compensated for.
Now the amplitude and phase error would be discussed in detail, which will deteriorate severely the range resolution. Suppose a modulation in the phase or amplitude of the LFM waveform can be modeled as perturbations $\varphi(t)$ and $A(t)$ in the transmitted and LO signals which are not flat in the total bandwidth. Besides, a direct current or low-frequency component $S_{\mathrm{L}}(t)$ will result owing to the sampling in our practical $\mathrm{THz}$ radar system. Thus, formula (12) becomes

$$
\begin{aligned}
S_{B}(t)= & A_{B} \exp \left\{2 \pi \left(\mu \tau t+12 f_{C 1} \tau(t)\right.\right. \\
& \left.\left.-\mu \tau^{2} / 2\right)\right\} \cdot A(t) \cdot \exp \{\Delta \varphi(t)\}+S_{\mathrm{L}}(t)
\end{aligned}
$$

where $A(t)=A_{\mathrm{LO}}(t) \cdot A_{T}(t-\tau)$ and $\Delta \varphi(t)=\varphi_{\mathrm{LO}}(t)-\varphi_{T}(t-\tau)$. In order to compensate for this degradation, some steps are taken in signal processing.
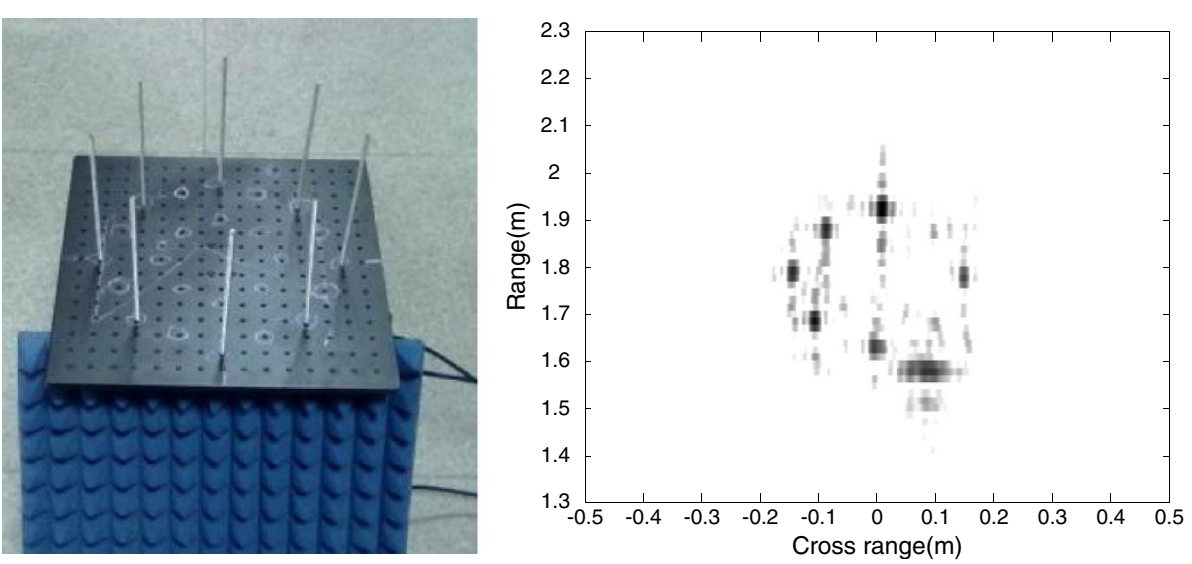

Figure 8 ISAR imaging for eight metal rods. 


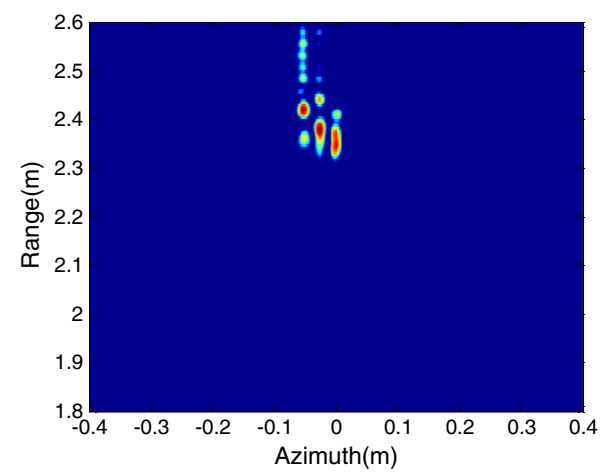

(a)

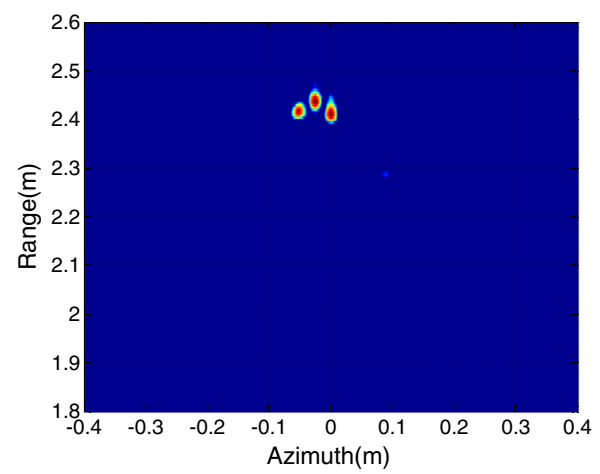

(b)

Figure 9 Imaging results of three metal spheres. (a) Imaging result before compensation. (b) Imaging result after compensation.

Firstly, a smoothing low-pass filter is applied to remove the low-frequency component $S_{\mathrm{L}}(t)$.

Secondly, a calibration signal is acquired as a reference using a typical bright target at a range $R 0$ in the radar's operating range, and the referent IF signal is recorded.

$$
S_{\text {ref }}(t)=\exp \left\{2 \pi\left(\mu \tau_{0} t\right)\right\} \cdot A_{R 0}(t) \cdot \exp \left\{\Delta \varphi_{R 0}(t)\right\}
$$

Finally, some other subsequent signals are divided by this calibration reference before spectral analysis, so the detected IF signal will be modified as

$$
S_{B}(t)=S_{B}(t) \cdot \exp \left\{-\Delta \varphi_{R 0}(t)\right\} / A_{R 0}(t)
$$

The signal spectrum will also be shifted by a known amount $\Delta f=\mu \tau_{0}=2 \mu R_{0} / c$ which can be added back easily. The resolution can improve significantly as long as the compensated amplitude approaches unity and the phase approaches zero.

The raw range spectrum of the two point-like targets is shown in Figure 4a. The main lobe of each target is severely broadened by the chirp waveform non-linearity, and we hardly recognize these two targets from this spectrogram. In contrast, Figure $4 \mathrm{~b}$ shows the same measurement where the signal is first divided by a previously acquired calibration waveform prior to calculating the range spectrum. After compensation, the broadened peaks become now much narrower. This process correctly removes the non-linearity effects and improves the range resolution of the radar system.

From the above formula derivation, we know that this method can perfectly compensate the range spectrum of the target which is located in the same distance with the reference target. Practically, calibrating using the target at $R=R_{0}$ results in excellent non-linearity compensation over the entire range of $R_{0} \pm 1$, and a high resolution can also be obtained.

\section{Experimental results}

As shown in Figure 5, the LFMCW radar prototyping system working in the $\mathrm{THz}$ waveband has been developed in the Radar Imaging Laboratory of UESTC. The transmitting signal bandwidth is $4.8 \mathrm{GHz}$.

In order to validate the performances of our radar system, some experiments have been conducted, and the results will be shown as follows. Figure 6 shows the experiment results of testing for metal ball range detection with a range of $1.25 \mathrm{~m}$, and detection of three metal balls can been seen in Figure 7. Finally, eight metal rods are arrayed in the platform which can rotate at a constant speed controlled by a servos system. Their image can be obtained by using an ISAR imaging method, as shown in Figure 8.

To verify the imaging performance of the non-linearity compensation, another experiment is performed, and the turntable system is used. The imaging results are shown in Figure 9. When the radar transmits the broadband signals, three metal spheres placed on the turntable are rotating at a constant speed. To achieve the same resolution in azimuth dimension, we can adjust the accumulating azimuth angle. In this experiment, this angle is $1.5^{\circ}$. As shown in Figure 9a, the accurate locations of the three spheres are not determined and the images of these spheres in range dimension are broadened. After compensation, the locations of the three spheres are corrected very well, as shown in Figure 9b.

\section{Conclusions}

It is feasible to use the LMFCW radar in THz waveband to obtain high range resolution and high-quality images. Some measures taken for correcting the signal error are valid in the intermediate frequency receiver to optimize the radar's range resolution. The radar's system structure low-noise LMF source can do the trick. In short, the $\mathrm{THz}$ LFMCW radar is an effective tool for range detection and imaging. 


\section{Competing interests}

The authors declare that they have no competing interests.

Received: 29 July 2013 Accepted: 14 November 2013

Published: 17 January 2014

\section{References}

1. J Li, YM Pi, Micro-doppler signature feature analysis in terahertz band. J. Infr. Mill. Terahertz. Waves. 31(3), 319-328 (2010)

2. JA Hejase, EJ Rothwell, $\mathrm{P}$ Chahal, A multiple angle method for $\mathrm{THz}$ time-domain material characterization. IEEE. Transac. Terahertz. Sci. Technol. 3(5), 656-665 (2013)

3. KB Cooper, RJ Dengler, N Llombart, B Thomas, G Chattopadhyay, PH Siegel: $\mathrm{THz}$ imaging radar for standoff personnel screening. IEEE. Trans. THz. Sci. Technol. 1(1), 169-182 (2011)

4. J Ding, M Kahl, O Loffeld, P Haring Bolívar, THz 3-D image formation using SAR techniques: simulation, processing and experimental results. IEEE. Transac. Terahertz. Sci. Technol. 3(5), 606-616 (2013)

5. J Moll, P Schöps, V Krozer, Towards three-dimensional millimeter-wave radar with the bistatic fast-factorized back-projection algorithm - potential and limitations. IEEE. Transac. Terahertz. Sci. Technol. 2(4), 432-440 (2012)

6. J Feng, Z Cao, Y Pi, Multiphase SAR image segmentation with $\mathrm{G} 0$ statistical model based active contours. IEEE. Trans. Geosci. Remote. Sens. 51(2), 4190-4199 (2013)

7. F Friederich, W von Spiegel, M Bauer, F Meng, MD Thomson, S Boppel, A Lisauskas, B Hils, V Krozer, A Keil, T Loffler, R Henneberger, AK Huhn, G Spickermann, PH Bolivar, HG Roskos: THz active imaging systems with realtime capabilities. IEEE. Trans. Terahertz. Technol. 1(1), 183-200 (2011)

8. M Pätzold, M Kahl, TA Keil, T Löffler, PH Bolívar, A Kolb, Simulation and data-processing framework for hybrid synthetic aperture THz systems including THz-scattering. IEEE. Transac. Terahertz. Sci. Technol. 3(5), 625-634 (2013)

9. P Meta, LP Hoogeboom, Ligthart, Range non-linearities correction in FMCW SAR, in Proc. IGARSS (Denver, CO, 2006), pp. 403-406

10. RJ Dengler, F Maiwald, PH Siegel, A compact $600 \mathrm{GHz}$ electronically tunable vector measurement system for submillimeter wave imaging, in IEEE MTT-S Int Digest (San Francisco, 2006), pp. 1923-1926

11. H Essen, A Wahlen, R Sommer, W Johannes, R Brauns, M Schlechtweg, A Tessmann, High-bandwidth $220 \mathrm{GHz}$ experimental radar. Electron. Lett. 43(20), 1114-1116 (2007)

12. RJ Dengler, KB Cooper, G Chattopadhyay, I Mehdi, E Schlecht, A Skalare, C Chen, PH Siegel, $600 \mathrm{GHz}$ imaging radar with $2 \mathrm{~cm}$ range resolution, in IEEE MTT-S Int Digest (Honolulu, 2007), pp. 137-1374

13. KB Cooper, RJ Dengler, G Chattopadhyay, E Schlecht, J Gill, A Skalare, I Mehdi, PH Siegel, A high-resolution imaging radar at $580 \mathrm{GHz}$. IEEE. Micro. Wire. Comp. Lett. 18(1), 64-66 (2008)

14. H Essen, S Stanko, R Sommer, A Wahlen, R Brauns, J Wilcke, W Johannes, A Tessmann, M Schlechtweg, A high performance 200-GHz broadband experimental radar. IEEE. 33rd. Int. Conf. Infr. Mill. Terahertz. Waves. IRMMW-THz. 2, 527-528 (2008)

doi:10.1186/1687-1499-2014-10

Cite this article as: Yao and Pi: Terahertz active imaging radar: preprocessing and experiment results. EURASIP Journal on Wireless Communications and Networking 2014 2014:10.

\section{Submit your manuscript to a SpringerOpen ${ }^{\circ}$ journal and benefit from:}

- Convenient online submission

- Rigorous peer review

- Immediate publication on acceptance

- Open access: articles freely available online

- High visibility within the field

- Retaining the copyright to your article

Submit your next manuscript at $>$ springeropen.com 